TITLE:

\title{
Slip/jump coefficients and Knudsen-layer corrections for the Shakhov model occurring in the generalized slip-flow theory
}

\author{
AUTHOR(S): \\ Hattori, Masanari; Takata, Shigeru
}

\section{CITATION:}

Hattori, Masanari ...[et al]. Slip/jump coefficients and Knudsen-layer corrections for the Shakhov model occurring in the generalized slip-flow theory. AlP Conference Proceedings 2019, 2132: 130012.

ISSUE DATE:

2019-08-05

URL:

http://hdl.handle.net/2433/243854

\section{RIGHT:}

This article may be downloaded for personal use only. Any other use requires prior permission of the author and AIP Publishing. This article appeared in Masanari Hattori and Shigeru Takata, AIP Conference Proceedings 2132, 130012 (2019) and may be found at https://doi.org/10.1063/1.5119632.; The full-text file will be made open to the public on 5 August 2020 in accordance with publisher's 'Terms and Conditions for Self-Archiving'. 


\title{
Slip/Jump Coefficients and Knudsen-Layer Corrections for the Shakhov Model Occurring in the Generalized Slip-Flow Theory
}

\author{
Masanari Hattori ${ }^{1}$ and Shigeru Takata ${ }^{1, a)}$ \\ ${ }^{1}$ Department of Aeronautics and Astronautics, Kyoto University, Kyoto 615-8540, Japan

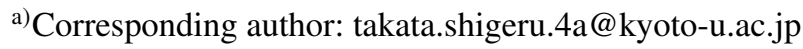

\begin{abstract}
The slip/jump coefficients and the Knudsen-layer functions for the time-dependent version of the generalized slip-flow theory have been obtained for the Shakhov model up to the second order of the Knudsen number expansion. Simple but exact conversion formulas from the Bhatnagar-Gross-Krook (BGK) model have also been established.
\end{abstract}

\section{INTRODUCTION}

Recently, in $[1,2,3]$, the authors have extended the generalized slip-flow theory for steady flows of slightly rarefied gases [4] to unsteady ones. In the theory, the system of fluid-dynamic-type equations and their appropriate slip/jump boundary conditions are explicitly obtained up to the second order in the Knudsen number expansion. The system describes the overall behavior of the gas. The corrections to its solution inside the Knudsen layer are obtained as well up to the second order. This extension was carried out for the linear case of the theory. The complete set of data required in applying the theory is available from Kyoto University Research Information Repository (http://hdl.handle.net/2433/199811). Currently, those data are obtained for the hard-sphere (HS) gas, the BhatnagarGross-Krook (BGK) model [5], and the Ellipsoidal-Statistical (ES) model [6] under the diffuse reflection condition. In the present paper, we prepare the corresponding data for the Shakhov model [7].

The Shakhov and ES models may be regarded as extensions of the celebrated BGK model, the relaxation model toward a local Maxwellian. Their advantage over the BGK model is that they can reproduce the correct Prandtl number of the gas. Since thermal phenomena and mechanical ones are mostly coupled in rarefied gases, this feature is advantageous. These models are now widely used as well in place of the BGK model. When the Knudsen number is small, the direct solution of these model equations becomes difficult. It is the slip-flow theory that can effectively complement the solution. With these in mind, the present paper aims at newly providing the slip/jump coefficients and the Knudsen-layer functions for the Shakhov model. The provided data will make it easier to access the generalized slip-flow theory.

In the Shakhov model, the velocity distribution function (VDF) is driven not to the local Maxwellian, but to its slight extension such that the heat flow is reproduced appropriately. This flexibility enables us to adjust the Prandtl number to realistic values. In the meantime, thanks to its similarity to the local Maxwellian, when the deviation from the local equilibrium is small, simple conversion formulas from the BGK model can be established. This fact is similar to the case of the ES model (see, e.g., [8, p. 319] and [9, 10, 11, 12]). In the present work, it is focused on and we discuss the conversion formulas which hold at the level of the VDF as well as the transport coefficient, the slip/jump coefficient, and the structure of the Knudsen layer.

\section{GENERALIZED SLIP-FLOW THEORY: A BRIEF SUMMARY}

According to the generalized slip-flow theory for unsteady systems [1,2,3], the behavior of a slightly rarefied gas with a small Reynolds number can be described in the bulk region by the Stokes-type set of equations and their

31st International Symposium on Rarefied Gas Dynamics

AIP Conf. Proc. 2132, 130012-1-130012-8; https://doi.org/10.1063/1.5119632

Published by AIP Publishing. 978-0-7354-1874-5/\$30.00 
slip/jump boundary conditions. In the Knudsen layer, this fluid-dynamic description (the fluid-dynamic part) needs to be corrected (the Knudsen-layer correction). The slip/jump boundary condition and the Knudsen-layer correction are required at the level of the first and higher orders of the Knudsen number.

Let us denote by $t_{0} t$ the time, by $L x_{i}$ the position, by $\rho_{0}(1+\omega), p_{0}(1+P), T_{0}(1+\tau)$, and $\left(2 R T_{0}\right)^{1 / 2} u_{i}$ the density, pressure, temperature, and velocity of the gas, respectively. The $\left(2 R T_{0}\right)^{1 / 2} u_{i \mathrm{w}}$ and $T_{0}\left(1+\tau_{\mathrm{w}}\right)$ denote the velocity and temperature of the boundary. Here $t_{0}$ is the reference time scale, $L$ is the characteristic length of the system, and $R$ is the specific gas constant. The $\rho_{0}, p_{0}$, and $T_{0}$ are the density, pressure, and temperature in the reference equilibrium state at rest $\left(p_{0}=\rho_{0} R T_{0}\right)$. We also denote by $n_{i}$ the unit normal vector to the boundary pointed to the gas and by $t_{i}$ an arbitrary unit tangential vector to the boundary. The Knudsen number $\mathrm{Kn}$ is defined as $\mathrm{Kn}=\ell_{0} / L$ with $\ell_{0}$ being the mean free path of a gas molecule in the reference equilibrium state at rest ${ }^{1}$. In the present paper, we use $\varepsilon$ defined as $\varepsilon=(\sqrt{\pi} / 2) \mathrm{Kn}$ instead of $\mathrm{Kn}$ in order to emphasize its smallness. In the above mentioned works [1, 2, 3], it is assumed that there is no external force and that the domain does not deform in time and thus $u_{i w} n_{i}=0$. Moreover, $t_{0}$ is taken as $t_{0}=L /\left[\varepsilon\left(2 R T_{0}\right)^{1 / 2}\right]$ based on the characteristic time of diffusive phenomena.

The fluid-dynamic part and the Knudsen-layer correction for each macroscopic quantity $h\left(h=\omega, P, \tau, u_{i}\right)$ are denoted by $h_{\mathrm{H}}$ and $h_{\mathrm{K}}$, respectively: $h=h_{\mathrm{H}}+h_{\mathrm{K}}$. The $h_{\mathrm{H}}$ and $h_{\mathrm{K}}$ are respectively expanded in a power series of $\varepsilon$ : $h_{\mathrm{H}}=h_{\mathrm{H} 0}+h_{\mathrm{H} 1} \varepsilon+h_{\mathrm{H} 2} \varepsilon^{2}+\cdots$ and $h_{\mathrm{K}}=h_{\mathrm{K} 1} \varepsilon+h_{\mathrm{K} 2} \varepsilon^{2}+\cdots$. Boundary data $u_{i \mathrm{w}}$ and $\tau_{\mathrm{w}}$ are expanded as well, e.g., $u_{i \mathrm{w}}=u_{i \mathrm{w} 0}+u_{i \mathrm{w} 1} \varepsilon+\cdots$. Note that the expansion of $h_{\mathrm{K}}$ starts from the first order of $\varepsilon$. Then, the above mentioned Stokes-type set of equations and the slip/jump boundary condition over smooth solid bodies are given as follows:

\section{Stokes-type set of equations}

$$
\begin{aligned}
& \partial_{i} P_{\mathrm{H} 0}=0, \\
& \partial_{t} \omega_{\mathrm{H} m-1}+\partial_{i} u_{i \mathrm{H} m}=0, \\
& \partial_{t} u_{i \mathrm{H} m}+\frac{1}{2} \partial_{i} P_{\mathrm{H} m+1}^{*}-\frac{1}{2} \gamma_{1} \Delta u_{i \mathrm{H} m}+\frac{1}{4}\left(\gamma_{1} \gamma_{10}-2 \gamma_{6}\right) \Delta^{2} u_{i \mathrm{H} m-2}=0, \\
& \partial_{t} \tau_{\mathrm{H} m}-\frac{2}{5} \partial_{t} P_{\mathrm{H} m}-\frac{1}{2} \gamma_{2} \Delta \tau_{\mathrm{H} m}+\frac{1}{10}\left(\gamma_{2} \gamma_{3}-\frac{13}{2} \gamma_{11}\right) \Delta^{2} \tau_{\mathrm{H} m-2}=0,
\end{aligned}
$$

where $\partial_{i}=\partial / \partial x_{i}, \partial_{t}=\partial / \partial t, \Delta=\partial_{j}^{2}$, and

$$
\begin{aligned}
& P_{\mathrm{H} m+1}^{*}=P_{\mathrm{H} m+1}-\frac{1}{6}\left(\gamma_{2} \gamma_{1}-4 \gamma_{3}\right) \Delta \tau_{\mathrm{H} m-1}+\frac{1}{5} \gamma_{1} \partial_{t} P_{\mathrm{H} m-1}, \\
& P_{\mathrm{H} m}=\omega_{\mathrm{H} m}+\tau_{\mathrm{H} m} .
\end{aligned}
$$

\section{Slip/jump boundary conditions}

$$
\begin{aligned}
u_{i \mathrm{H} m} n_{i}= & a_{1}^{(1)} \partial_{i} \overline{\partial_{j} u_{k \mathrm{H} m-2}} n_{i} n_{j} n_{k}+a_{2}^{(1)}\left(2 \bar{\kappa} \partial_{i} \tau_{\mathrm{H} m-2} n_{i}-\Delta \tau_{\mathrm{H} m-2}+\partial_{i} \partial_{j} \tau_{\mathrm{H} m-2} n_{i} n_{j}\right), \\
u_{i \mathrm{H} m} t_{i}= & u_{i \mathrm{w} m} t_{i}+b_{1}^{(1)} \overline{\partial_{i} u_{j \mathrm{H} m-1}} n_{i} t_{j}+b_{2}^{(1)} \partial_{i} \tau_{\mathrm{H} m-1} t_{i}+b_{3}^{(1)} \partial_{i} \partial_{j} \tau_{\mathrm{H} m-2} n_{i} t_{j}+b_{4}^{(1)} \partial_{i} \overline{\partial_{j} u_{k \mathrm{H} m-2}} n_{i} n_{j} t_{k} \\
& +b_{5}^{(1)} \bar{\kappa} \overline{\partial_{j} u_{k \mathrm{H} m-2}} n_{j} t_{k}+b_{6}^{(1)} \kappa_{i j} \overline{\partial_{j} u_{k \mathrm{H} m-2}} n_{k} t_{i}+b_{7}^{(1)} \kappa_{i j} \partial_{i} \tau_{\mathrm{H} m-2} t_{j}+b_{8}^{(1)} \bar{\kappa} \partial_{i} \tau_{\mathrm{H} m-2} t_{i}, \\
\tau_{\mathrm{H} m}= & \tau_{\mathrm{w} m}+c_{1}^{(0)} \partial_{i} \tau_{\mathrm{H} m-1} n_{i}-c_{2}^{(0)} \partial_{i} \partial_{j} \tau_{\mathrm{H} m-2} n_{i} n_{j}+c_{3}^{(0)} \partial_{i} \overline{\partial_{j} u_{k \mathrm{H} m-2}} n_{i} n_{j} n_{k}+c_{4}^{(0)} \bar{\kappa} \partial_{i} \tau_{\mathrm{H} m-2} n_{i} \\
& +c_{5}^{(0)} \partial_{i} u_{i \mathrm{H} m-1}+\left(c_{6}^{(0)}+c_{2}^{(0)}\right) \Delta \tau_{\mathrm{H} m-2} .
\end{aligned}
$$

Here $m=0,1,2$ and $h_{\mathrm{H}-1}$ and $h_{\mathrm{H}-2}$ should be read as zero. This convention also applies to formulas of the Knudsenlayer corrections (3). The $\gamma$ 's in (1) are dimensionless transport coefficients in the reference equilibrium state, which depend on the gas model. Among them, $\gamma_{1}$ and $\gamma_{2}$ are the dimensionless viscosity and thermal conductivity, respectively. Their ratio is the Prandtl number: $\operatorname{Pr}=\gamma_{1} / \gamma_{2} .{ }^{2}$ In (2), $a$ 's, $b$ 's, and $c$ 's are the slip/jump coefficients, which depend on gas-surface interaction models. $\bar{f}_{i j}=f_{i j}+f_{j i}-(2 / 3) f_{k k} \delta_{i j}$, where $\delta_{i j}$ is the Kronecker delta. $\bar{\kappa}=\left(\kappa_{1}+\kappa_{2}\right) / 2$,

\footnotetext{
${ }^{1}$ For example, $L$ can be taken to be the diameter of a pipe for pipe flow and that of a sphere for flow past the sphere, respectively. In the former, $\rho_{0}$ and $T_{0}$ can be taken to be the average gas density and temperature of the pipe. In the latter, they can be taken to be the density and temperature at a far field.

${ }^{2}$ The viscosity $\mu(T)$ is a positive function of temperature $T$. At the reference state, it is given by $\mu\left(T_{0}\right)=(\sqrt{\pi} / 2) \gamma_{1} p_{0}\left(2 R T_{0}\right)^{-1 / 2} \ell_{0}$.
} 
$\kappa_{i j}=\kappa_{1} \ell_{i} \ell_{j}+\kappa_{2} m_{i} m_{j}$, where $\kappa_{1} / L$ and $\kappa_{2} / L$ are the two principal curvatures of the boundary. The $\kappa_{1}$ and $\kappa_{2}$ are taken negative when the corresponding center of the curvature lies on the side of the gas. The $\ell_{i}$ and $m_{i}$ are the direction cosines of the principal directions corresponding to $\kappa_{1}$ and $\kappa_{2}$, respectively. The behavior of the gas in the bulk region is determined by (1) and (2). To be more specific, by (1a) the pressure is uniform at the leading order. By (1b)-(1d) and the boundary conditions (2) for $m=0$ the leading-order flow velocity and temperature are determined with the first-order pressure. By (1b)-(1d) and the boundary conditions (2) for $m=1$ the first-order flow velocity and temperature are determined with the second-order pressure, and so on. Note that (2) for $m=0$ is just the no-slip/no-jump condition.

Knudsen-layer correction Once the fluid-dynamic part is obtained by the above process, the correction to this part near the boundary (the Knudsen-layer correction) is given by the following formulas:

$$
\begin{aligned}
u_{i \mathrm{~K} m} n_{i}= & -\int_{\eta}^{\infty} Y_{2}^{(1)}(z) d z\left(2 \bar{\kappa} \partial_{i} \tau_{\mathrm{H} m-2} n_{i}-\Delta \tau_{\mathrm{H} m-2}+\partial_{i} \partial_{j} \tau_{\mathrm{H} m-2} n_{i} n_{j}\right)-\frac{1}{2} \int_{\eta}^{\infty} Y_{1}^{(1)}(z) d z \partial_{i} \overline{\partial_{j} u_{k \mathrm{H} m-2}} n_{i} n_{j} n_{k}, \\
u_{i \mathrm{~K} m} t_{i}= & Y_{1}^{(1)}(\eta) \overline{\partial_{i} u_{j \mathrm{H} m-1}} n_{i} t_{j}+Y_{2}^{(1)}(\eta) \partial_{i} \tau_{\mathrm{H} m-1} t_{i}+Y_{3}^{(1)}(\eta) \partial_{i} \partial_{j} \tau_{\mathrm{H} m-2} n_{i} t_{j}+Y_{4}^{(1)}(\eta) \partial_{i} \overline{\partial_{j} u_{k \mathrm{H} m-2}} n_{i} n_{j} t_{k} \\
& +Y_{5}^{(1)}(\eta) \bar{\kappa} \overline{\partial_{j} u_{k \mathrm{H} m-2}} n_{j} t_{k}+Y_{6}^{(1)}(\eta) \kappa_{i j} \overline{\partial_{j} u_{k \mathrm{H} m-2}} n_{k} t_{i}+Y_{7}^{(1)}(\eta) \kappa_{i j} \partial_{i} \tau_{\mathrm{H} m-2} t_{j}+Y_{8}^{(1)}(\eta) \bar{\kappa} \partial_{i} \tau_{\mathrm{H} m-2} t_{i}, \\
{\left[\begin{array}{c}
\omega_{\mathrm{K} m} \\
\tau_{\mathrm{K} m}
\end{array}\right]=} & {\left[\begin{array}{c}
\Omega_{1}^{(0)}(\eta) \\
\Theta_{1}^{(0)}(\eta)
\end{array}\right] \partial_{i} \tau_{\mathrm{H} m-1} n_{i}-\left[\begin{array}{c}
\Omega_{2}^{(0)}(\eta) \\
\Theta_{2}^{(0)}(\eta)
\end{array}\right] \partial_{i} \partial_{j} \tau_{\mathrm{H} m-2} n_{i} n_{j}+\left[\begin{array}{c}
\Omega_{3}^{(0)}(\eta) \\
\Theta_{3}^{(0)}(\eta)
\end{array}\right] \partial_{i} \overline{\partial_{j} u_{k \mathrm{H} m-2}} n_{i} n_{j} n_{k} } \\
& +\left[\begin{array}{c}
\Omega_{4}^{(0)}(\eta) \\
\Theta_{4}^{(0)}(\eta)
\end{array}\right] \bar{\kappa} \partial_{i} \tau_{\mathrm{H} m-2} n_{i}+\left[\begin{array}{c}
\Omega_{6}^{(0)}(\eta)+\Omega_{2}^{(0)}(\eta) \\
\Theta_{6}^{(0)}(\eta)+\Theta_{2}^{(0)}(\eta)
\end{array}\right] \Delta \tau_{\mathrm{H} m-2}+\left[\begin{array}{c}
\Omega_{5}^{(0)}(\eta) \\
\Theta_{5}^{(0)}(\eta)
\end{array}\right] \partial_{i} u_{i \mathrm{H} m-1} .
\end{aligned}
$$

Here, $\Omega$ 's, $\Theta$ 's, and $Y$ 's are elemental functions, which we call the Knudsen-layer functions. The $\eta$ is the stretched coordinate normal to the boundary defined by $x_{i}=x_{\mathrm{w} i}+\varepsilon \eta n_{i}$, where $L x_{\mathrm{w} i}$ is the position on the boundary. Note that the quantities with the subscript $\mathrm{H}$ represent their value on the boundary $x_{i}=x_{\mathrm{w} i}$ (or $\eta=0$ ). Because there is no net flow across the solid surface, $a$ 's in (2) are related to $Y^{\prime}$ s as $a_{1}^{(1)}=(1 / 2) \int_{0}^{\infty} Y_{1}^{(1)}(z) d z$ and $a_{2}^{(1)}=\int_{0}^{\infty} Y_{2}^{(1)}(z) d z$. Further detailed information is available from $[1,2,3]$.

Elemental half-space problems In deriving (2) and (3), the locally isotropic property [4, 1] of the kinetic boundary condition is assumed. The Knudsen-layer functions $\Omega$ 's and $\Theta$ 's (or $Y$ 's) are expressed as moments of the elemental solutions to the half-space problems shown below. They are of the form $\phi_{j}^{(0)}\left(\eta, \zeta_{n}, \zeta\right)(j=1,2, \ldots, 6)[$ or $\left.\phi_{j}^{(1)}\left(\eta, \zeta_{n}, \zeta\right)(j=1,2, \ldots, 8)\right]$, where $\zeta=|\zeta|=\left(\zeta_{i}^{2}\right)^{1 / 2}$ and $\zeta_{n}=\zeta_{i} n_{i}$ with $\left(2 R T_{0}\right)^{1 / 2} \zeta$ being the molecular velocity. The jump coefficients $c_{j}^{(0)}$ [or the slip coefficients $b_{j}^{(1)}$ ] are determined simultaneously with these solutions.

Elemental problems for $\phi_{j}^{(0)}\left(\eta, \zeta_{n}, \zeta\right)(j=1,2, \ldots, 6)$ :

$$
\begin{aligned}
\zeta_{n} \partial_{\eta} \phi_{j}^{(0)} & =\mathcal{L}\left(\phi_{j}^{(0)}\right)-I_{j}^{(0)}, \\
\phi_{j}^{(0)} & =-\widetilde{\mathcal{K}}\left(\zeta^{2}\right) c_{j}^{(0)}+\mathcal{K}\left(\phi_{j}^{(0)}\right)+\widetilde{\mathcal{K}}\left(g_{j}^{(0)}\right), \quad \zeta_{n}>0, \eta=0, \\
\phi_{j}^{(0)} & \rightarrow 0 \quad \text { as } \quad \eta \rightarrow \infty,
\end{aligned}
$$

where $\mathcal{L}$ is the linearized collision integral operator, $\mathcal{K}$ is the linearized scattering operator, which admits the functional form of $\phi$ 's with respect to the molecular velocity, and $\widetilde{\mathcal{K}}=f-\mathcal{K}(f)$. The $I^{(0)}$ 's and $g^{(0)}$ 's are given by

$$
\begin{aligned}
& I_{1}^{(0)}=0, g_{1}^{(0)}=\zeta_{n} A, \quad I_{2}^{(0)}=\frac{1}{2}\left(\zeta^{2}-\zeta_{n}^{2}\right) \phi_{2}^{(1)}, g_{2}^{(0)}=2 a_{2}^{(1)} \zeta_{n}+\frac{1}{2}\left(\zeta^{2}-3 \zeta_{n}^{2}\right)\left(b_{2}^{(1)} B+F\right), \\
& I_{3}^{(0)}=-\frac{1}{4}\left(\zeta^{2}-\zeta_{n}^{2}\right) \phi_{1}^{(1)}, g_{3}^{(0)}=-2 a_{1}^{(1)} \zeta_{n}-\frac{1}{4} b_{1}^{(1)}\left(\zeta^{2}-3 \zeta_{n}^{2}\right) B-\frac{1}{2} \zeta_{n}\left(D_{1}-\left(\zeta^{2}-2 \zeta_{n}^{2}\right) D_{2}\right), \\
& I_{4}^{(0)}=\left(\zeta^{2}-\zeta_{n}^{2}\right)\left(\partial_{\zeta_{n}} \phi_{1}^{(0)}-\phi_{2}^{(1)}\right), g_{4}^{(0)}=-4 a_{2}^{(1)} \zeta_{n}-b_{2}^{(1)}\left(\zeta^{2}-3 \zeta_{n}^{2}\right) B, \\
& I_{5}^{(0)}=0, g_{5}^{(0)}=-\frac{1}{3}\left(\zeta^{2}-3 \zeta_{n}^{2}\right) B, \quad I_{6}^{(0)}=0, g_{6}^{(0)}=-\frac{1}{3}\left(\zeta^{2}-3 \zeta_{n}^{2}\right) F+F_{d} .
\end{aligned}
$$


Elemental problems for $\phi_{j}^{(1)}\left(\eta, \zeta_{n}, \zeta\right)(j=1,2, \ldots, 8)$ :

$$
\begin{aligned}
\zeta_{n} \partial_{\eta} \zeta_{t} \phi_{j}^{(1)} & =\mathcal{L}\left(\zeta_{t} \phi_{j}^{(1)}\right)-\zeta_{t} I_{j}^{(1)}, \\
\zeta_{t} \phi_{j}^{(1)} & =-2 \widetilde{\mathcal{K}}\left(\zeta_{t}\right) b_{j}^{(1)}+\mathcal{K}\left(\zeta_{t} \phi_{j}^{(1)}\right)+\widetilde{\mathcal{K}}\left(\zeta_{t} g_{j}^{(1)}\right), \quad \zeta_{n}>0, \eta=0, \\
\zeta_{t} \phi_{j}^{(1)} & \rightarrow 0 \quad \text { as } \quad \eta \rightarrow \infty,
\end{aligned}
$$

where $\zeta_{t}=\zeta_{i} t_{i}$ and

$$
\begin{aligned}
& I_{1}^{(1)}=0, g_{1}^{(1)}=\zeta_{n} B, \quad I_{2}^{(1)}=0, g_{2}^{(1)}=A, \quad I_{3}^{(1)}=\phi_{1}^{(0)}, g_{3}^{(1)}=2 \zeta_{n} F, \quad I_{4}^{(1)}=0, g_{4}^{(1)}=-\left(D_{1}+\zeta_{n}^{2} D_{2}\right), \\
& I_{5}^{(1)}=\frac{1}{2}\left(\zeta^{2}-\zeta_{n}^{2}\right) \partial_{\zeta_{n}} \phi_{1}^{(1)}, g_{5}^{(1)}=-2 D_{1}-\frac{1}{2}\left(\zeta^{2}-\zeta_{n}^{2}\right) D_{2}, \quad I_{6}^{(1)}=\frac{1}{2} \partial_{\zeta_{n}}\left(\left(\zeta^{2}-\zeta_{n}^{2}\right) \phi_{1}^{(1)}\right), g_{6}^{(1)}=g_{5}^{(1)}-g_{4}^{(1)}, \\
& I_{7}^{(1)}=\frac{1}{2} \partial_{\zeta_{n}}\left(\left(\zeta^{2}-\zeta_{n}^{2}\right) \phi_{2}^{(1)}\right)+\phi_{1}^{(0)}, g_{7}^{(1)}=0, \quad I_{8}^{(1)}=\frac{1}{2}\left(\zeta^{2}-\zeta_{n}^{2}\right) \partial_{\zeta_{n}} \phi_{2}^{(1)}, g_{8}^{(1)}=0 .
\end{aligned}
$$

Note that $I$ 's and $g$ 's above are given sources in the equations and the boundary conditions. The functions $A, B, \ldots$, and $F_{d}$ appearing in the expression of $g$ 's are functions of $\zeta$. They are defined as the solution of the following integral equations:

$$
\begin{aligned}
& \mathcal{L}\left(\zeta_{i} A\right)=-\zeta_{i}\left(\zeta^{2}-\frac{5}{2}\right) \quad \text { with }\left\langle\zeta^{2} A\right\rangle=0, \quad \mathcal{L}\left(\zeta_{i j} B\right)=-2 \zeta_{i j}, \quad \mathcal{L}\left(\zeta_{i j} F\right)=\zeta_{i j} A, \\
& \mathcal{L}\left[\left(\zeta_{i} \delta_{j k}+\zeta_{j} \delta_{i k}+\zeta_{k} \delta_{i j}\right) D_{1}+\zeta_{i} \zeta_{j} \zeta_{k} D_{2}\right]=\gamma_{1}\left(\zeta_{i} \delta_{j k}+\zeta_{j} \delta_{i k}+\zeta_{k} \delta_{i j}\right)-\zeta_{i} \zeta_{j} \zeta_{k} B \quad \text { with }\left\langle\zeta^{2}\left(5 D_{1}+\zeta^{2} D_{2}\right)\right\rangle=0, \\
& \mathcal{L}\left(F_{d}\right)=-\frac{5}{6} \gamma_{2}\left(\zeta^{2}-\frac{3}{2}\right)+\frac{1}{3} \zeta^{2} A \quad \text { with }\left\langle F_{d}\right\rangle=\left\langle\zeta^{2} F_{d}\right\rangle=0,
\end{aligned}
$$

where $\zeta_{i j}=\zeta_{i} \zeta_{j}-(1 / 3) \zeta^{2} \delta_{i j},\langle f\rangle \equiv \int f E(\zeta) d \zeta$, and $E(z)=\pi^{-3 / 2} \exp \left(-z^{2}\right)$. The $\gamma^{\prime}$ s in (1) are expressed in terms of these functions as

$$
\begin{aligned}
& \gamma_{1}=\frac{2}{15}\left\langle\zeta^{4} B\right\rangle, \quad \gamma_{2}=\frac{4}{15}\left\langle\zeta^{4} A\right\rangle, \quad \gamma_{3}=\frac{2}{15}\left\langle\zeta^{4} A B\right\rangle, \quad \gamma_{6}=\frac{1}{15}\left\langle\zeta^{4} B D_{1}\right\rangle+\frac{1}{35}\left\langle\zeta^{6} B D_{2}\right\rangle, \\
& \gamma_{10}=\frac{1}{15}\left\langle\zeta^{4} B^{2}\right\rangle, \quad \gamma_{11}=-\frac{2}{39}\left(\gamma_{2}\left(2\left\langle\zeta^{2} A^{2}\right\rangle+\gamma_{3}\right)+\frac{16}{15}\left\langle\zeta^{4} A F\right\rangle+4\left\langle\zeta^{2} A F_{d}\right\rangle\right) .
\end{aligned}
$$

Once $\phi$ 's are obtained, the Knudsen-layer functions are obtained as their moments:

$$
\Omega_{j}^{(0)}=\left\langle\phi_{j}^{(0)}\right\rangle, \quad \Theta_{j}^{(0)}=\frac{2}{3}\left\langle\left(\zeta^{2}-\frac{3}{2}\right) \phi_{j}^{(0)}\right\rangle, \quad Y_{j}^{(1)}=\frac{1}{2}\left\langle\left(\zeta^{2}-\zeta_{n}^{2}\right) \phi_{j}^{(1)}\right\rangle .
$$

Finally, from the energy conservation law of (4a), we obtain the following equalities, which are used later in the reduction to the BGK model:

$$
\left\langle\zeta_{n}\left(\zeta^{2}-\frac{5}{2}\right) \phi_{2}^{(0)}\right\rangle=\int_{\eta}^{\infty} H_{2}^{(1)}(z) d z, \quad\left\langle\zeta_{n}\left(\zeta^{2}-\frac{5}{2}\right) \phi_{4}^{(0)}\right\rangle=-2 \int_{\eta}^{\infty} H_{2}^{(1)}(z) d z, \quad\left\langle\zeta_{n}\left(\zeta^{2}-\frac{5}{2}\right) \phi_{\alpha}^{(0)}\right\rangle=0, \quad(\alpha=1,5,6) .
$$

\section{SHAKHOV MODEL AND ITS RELATION TO THE BGK MODEL}

\section{Preparation}

Denoting by $\tilde{t}$ the time, by $X_{i}$ the spatial coordinates, by $\xi_{i}$ the molecular velocity, and by $f$ the VDF, the original Boltzmann equation without external force can be symbolically written as

$$
\frac{\partial f}{\partial \tilde{t}}+\xi_{i} \frac{\partial f}{\partial X_{i}}=Q(f)
$$

The Shakhov model is defined by setting the collision integral $Q(f)$ as follows: [7]

$$
Q(f)=Q_{\mathrm{Sh}}(f)=A_{c}(T) \rho\left\{\mathcal{M}(f)\left[1+\frac{2}{5}(1-\operatorname{Pr}) \frac{q_{i}\left(\xi_{i}-v_{i}\right)}{p R T}\left(\frac{\left(\xi_{j}-v_{j}\right)^{2}}{2 R T}-\frac{5}{2}\right)\right]-f\right\},
$$




$$
A_{c}(T)=\frac{R T}{\mu(T)}, \quad \mathcal{M}(f)=\frac{\rho}{(2 \pi R T)^{3 / 2}} \exp \left(-\frac{\left(\xi_{i}-v_{i}\right)^{2}}{2 R T}\right) .
$$

Here $\rho, v_{i}, T, p, q_{i}$, and $\mu(T)$ are respectively the density, flow velocity, temperature, pressure, heat-flow vector, and viscosity of the gas. The Pr is the Prandtl number of the gas, which is an adjustable parameter in this model. For the Shakhov model, neither the positivity of the VDF nor the H theorem has been proven. Note, however, that when the deviation from the reference equilibrium is so small that linearization is allowed, the model satisfies the $\mathrm{H}$ theorem [7]. When $\operatorname{Pr}=1, Q_{\mathrm{Sh}}$ is reduced to the collision integral $Q_{\mathrm{BGK}}$ for the BGK model: ${ }^{3}$

$$
\left.Q_{\mathrm{Sh}}(f)\right|_{\mathrm{Pr}=1}=Q_{\mathrm{BGK}}(f)=A_{c}(T) \rho[\mathcal{M}(f)-f] .
$$

Hereinafter subscripts Sh, BGK, ES, and HS are used also for other quantities to indicate the model.

By setting $\ell_{0}=\left(8 R T_{0} / \pi\right)^{1 / 2} / A_{c}\left(T_{0}\right) \rho_{0}, \mathcal{L}$ in (4) and (5) for the Shakhov model is given as

$$
\begin{aligned}
& \mathcal{L}(\phi)=\mathcal{L}_{\mathrm{Sh}}(\phi)=\mathcal{L}_{\mathrm{BGK}}(\phi)+\frac{4}{5}(1-\operatorname{Pr}) \zeta_{i}\left(\zeta^{2}-\frac{5}{2}\right)\left\langle\zeta_{i}\left(\zeta^{2}-\frac{5}{2}\right) \phi\right\rangle, \\
& \mathcal{L}_{\mathrm{BGK}}(\phi)=\langle\phi\rangle+2 \zeta_{i}\left\langle\zeta_{i} \phi\right\rangle+\left\langle\frac{2}{3}\left(\zeta^{2}-\frac{3}{2}\right) \phi\right\rangle\left(\zeta^{2}-\frac{3}{2}\right)-\phi .
\end{aligned}
$$

Here $\mathcal{L}_{\mathrm{BGK}}$ is the linearized collision integral operator for the BGK model. As is seen from (7), even in the case $\operatorname{Pr} \neq 1$, $\mathcal{L}_{\mathrm{Sh}}$ and $\mathcal{L}_{\mathrm{BGK}}$ are different only when $\left\langle\zeta_{i}\left(\zeta^{2}-5 / 2\right) \phi\right\rangle \neq 0$. When it vanishes or is a given function in a specific form, the solutions for the Shakhov model can be recovered from those for the BGK model, as in the case of the ES model (see, e.g., $[8,9,10,11,12]$ ). This will be the clue in the discussion in the next subsection.

Before proceeding to details, we summarize the explicit form of the functions $A, B, \ldots$, and $F_{d}$ and the dimensionless transport coefficients $\gamma$ 's for the Shakhov model:

$$
\begin{aligned}
& A=\frac{1}{\operatorname{Pr}}\left(\zeta^{2}-\frac{5}{2}\right), \quad B=2, \quad D_{1}=-1+\frac{2(1-\operatorname{Pr})}{5 \operatorname{Pr}}\left(\zeta^{2}-\frac{5}{2}\right), \quad D_{2}=2, \quad F=-A, \quad F_{d}=-\frac{1}{3 \operatorname{Pr}}\left(\zeta^{4}-5 \zeta^{2}+\frac{15}{4}\right), \\
& \gamma_{1}=1, \quad \gamma_{2}=\frac{1}{\operatorname{Pr}}, \quad \gamma_{3}=\frac{1}{\operatorname{Pr}}, \quad \gamma_{6}=1+\frac{1-\operatorname{Pr}}{5 \operatorname{Pr}}, \quad \gamma_{10}=1, \quad \gamma_{11}=\frac{1}{\operatorname{Pr}^{3}}\left[1-\frac{18}{13}(1-\operatorname{Pr})\right] .
\end{aligned}
$$

They can be easily obtained, thanks to the simple form of $\mathcal{L}_{\mathrm{Sh}}$. Those for the BGK model are obtained by setting $\operatorname{Pr}=1$ in (8).

In the case of the HS gas and the ES model, $\ell_{0 \mathrm{HS}}=\left(\sqrt{2} \pi d_{m}^{2} \rho_{0} / m\right)^{-1}$ with $m$ and $d_{m}$ being the mass and diameter of a molecule and $\ell_{0 \mathrm{ES}}=\left(8 R T_{0} / \pi\right)^{1 / 2} \mu\left(T_{0}\right) / \mathrm{Pr}_{\mathrm{ES}} p_{0}$, respectively. The functions $A, B, \ldots$, and $F_{d}$ take different form from (8a) depending on the model, and $\gamma$ 's take the following values:

$$
\begin{aligned}
& \gamma_{1 \mathrm{HS}}=1.270042427, \quad \gamma_{2 \mathrm{HS}}=1.922284066, \quad\left(\operatorname{Pr}_{\mathrm{HS}}=\gamma_{1 \mathrm{HS}} / \gamma_{2 \mathrm{HS}}=0.6606944569\right), \quad \gamma_{3 \mathrm{HS}}=1.947906335, \\
& \gamma_{6 \mathrm{HS}}=1.419423836, \quad \gamma_{10 \mathrm{HS}}=1.63607346, \quad \gamma_{11 \mathrm{HS}}=2.7931173, \\
& \gamma_{1 \mathrm{ES}}=\operatorname{Pr}_{\mathrm{ES}}, \quad \gamma_{2 \mathrm{ES}}=1, \quad \gamma_{3 \mathrm{ES}}=\operatorname{Pr}_{\mathrm{ES}}, \quad \gamma_{6 \mathrm{ES}}=\operatorname{Pr}_{\mathrm{ES}}^{2}, \quad \gamma_{10 \mathrm{ES}}=\operatorname{Pr}_{\mathrm{ES}}^{2}, \quad \gamma_{11 \mathrm{ES}}=1+\frac{2}{13}\left(\operatorname{Pr}_{\mathrm{ES}}-1\right) .
\end{aligned}
$$

In comparing the data based on different collision models, it is common in the literature to take the viscosity or the thermal conductivity as a reference quantity, namely, to take $\gamma_{1} \ell_{0}$ or $\gamma_{2} \ell_{0}$ in common. For example, the conversion rule for $\ell_{0}$ between the Shakhov model and the HS gas is given as $\ell_{0 \mathrm{Sh}}=1.270042427 \ell_{0 \mathrm{HS}} \operatorname{or~Pr}_{\mathrm{Sh}}^{-1} \ell_{0 \mathrm{Sh}}=1.922284066 \ell_{0 \mathrm{HS}}$, leading to $\varepsilon_{\mathrm{Sh}}=1.270042427 \varepsilon_{\mathrm{HS}}$ or $\operatorname{Pr}_{\mathrm{Sh}}^{-1} \varepsilon_{\mathrm{Sh}}=1.922284066 \varepsilon_{\mathrm{HS}}$. Since $\operatorname{Pr}_{\mathrm{HS}}=0.6606944569$ is close to $2 / 3$, the viscosity-based conversion is practically equivalent to the thermal conductivity-based one between the Shakhov model with $\mathrm{Pr}_{\mathrm{Sh}}=2 / 3$ and the HS gas. This is the advantage of the Shakhov model over the BGK model.

\section{Reduction to the BGK Model}

In the present subsection, we discuss the feasibility of reduction of the problems (4) and (5) for the Shakhov model to those for the BGK model. At first, let us consider the problem (5). Thanks to the parity of $\zeta_{t} \phi_{j}^{(1)}$, the normal component

\footnotetext{
${ }^{3}$ In the original BGK model, $A_{c}$ is a positive constant because it is a simplified model for the pseudo-Maxwell molecular gas. Here, $A_{c}$ is extended to be a function of the temperature. The extension does not make a difference from the original under the linearized situation.
} 
of the heat-flow vector vanishes. However, the tangential component does not. Therefore simple conversion between $\mathcal{L}_{\mathrm{Sh}}\left[\zeta_{t} \phi_{j}^{(1)}\right]$ and $\mathcal{L}_{\mathrm{BGK}}\left[\zeta_{t} \phi_{j}^{(1)}\right]$ is not expected, while the desired conversion is possible for many elements for the problem (4). We will explain this below.

Thanks to the parity of $\phi_{j}^{(0)}$, the tangential component of the heat-flow vector vanishes. As for the normal component, it vanishes for $\phi_{1}^{(0)}, \phi_{5}^{(0)}$, and $\phi_{6}^{(0)}$, thanks to (6) (see [1, Appendix G.1]). Therefore, $\mathcal{L}_{\mathrm{Sh}}$ reduces to $\mathcal{L}_{\mathrm{BGK}}$ in the elemental problems for $\phi_{1}^{(0)}, \phi_{5}^{(0)}$, and $\phi_{6}^{(0)}$. Then taking into account the similarity of $I_{j}^{(0)}$ and $g_{j}^{(0)}$ between the two models, following simple conversions are obtained:

$$
\phi_{j \mathrm{Sh}}^{(0)}=\operatorname{Pr}^{-1} \phi_{j \mathrm{BGK}}^{(0)}(j=1,6), \quad \phi_{5 \mathrm{Sh}}^{(0)}=\phi_{5 \mathrm{BGK}}^{(0)} .
$$

The corresponding jump coefficients and the Knudsen-layer functions obey the same conversion.

Similar conversions do not hold for $\phi_{2}^{(0)}$ and $\phi_{4}^{(0)}$ separately. However, for the sum $\phi_{24}^{(0)} \equiv 2 \phi_{2}^{(0)}+\phi_{4}^{(0)}$, it holds that $\mathcal{L}_{\mathrm{Sh}}\left[\phi_{24}^{(0)}\right]=\mathcal{L}_{\mathrm{BGK}}\left[\phi_{24}^{(0)}\right]$. Consequently, the following conversion is obtained:

$$
\phi_{24 \mathrm{Sh}}^{(0)}=\operatorname{Pr}^{-1} \phi_{24 \mathrm{BGK}}^{(0)} .
$$

Here, in the above equation, (10a) for $j=1$ and the relation $F_{\mathrm{Sh}}=\operatorname{Pr}^{-1} F_{\mathrm{BGK}}$ are used. The corresponding jump coefficients and the Knudsen-layer functions again obey the same conversion.

In summary, to obtain the slip coefficients and the related Knudsen-layer functions we have to solve the problems (5) for the Shakhov model directly. As for the remaining problems (4), we have to solve only those for $\phi_{2}^{(0)}$ and $\phi_{3}^{(0)}$. The others can be obtained by the simple conversion rule from the data for the BGK model, if they are available. The conversion rule holds for arbitrary Prandtl number. Moreover, it holds for any locally isotropic boundary conditions supposed in [1], which include the diffuse reflection boundary condition as a special case. In the next section, we will show the results for $\operatorname{Pr}=2 / 3$ and the diffuse reflection boundary condition. Note that the values of $b_{1}^{(1)}, b_{2}^{(1)}$, and $c_{1}^{(0)}$ can be found in $[13,14,15]$ as well. The values in $[13,14,15]$ are very close to our results, though the data of the associated Knudsen-layer functions are not available from these references.

Incidentally, once $\phi_{1}^{(0)}, \phi_{1}^{(1)}$, and $\phi_{2}^{(1)}$ are obtained, all the slip/jump coefficients can be obtained by making use of the symmetry relation [16] as is done in [1]. Thus, as far as the slip/jump coefficients are concerned, newly required computations for the Shakhov model are only those for $\phi_{1}^{(1)}$ and $\phi_{2}^{(1)}$.

\section{NUMERICAL RESULTS}

As in the case of the BGK model, the problem for the Shakhov model can be converted to integral equations for the macroscopic quantities (see, e.g., [17] and [4, Appendix A.4]). In the meantime, the authors have already constructed an accurate direct numerical method [2,3] for the original formalism (4) and (5), which can safely handle the localized divergent singularity emerging at the level of the VDFs (see [3] for details). Here, the results obtained by the latter method is presented. Due to the limitation of space, here we show the slip/jump coefficients and a part of the Knudsenlayer functions for the diffuse reflection condition and $\operatorname{Pr}=2 / 3$. More detailed information (VDFs and tables and raw data of the Knudsen-layer functions) will be uploaded on Kyoto University Research Information Repository (http://hdl.handle.net/2433/199811).

Table 1 shows the values of the slip/jump coefficients $b_{1}^{(1)}, \ldots, b_{8}^{(1)}, c_{2}^{(0)}, c_{3}^{(0)}, a_{1}^{(1)}$, and $a_{2}^{(1)}$ for which new computations are required. The table includes those for the BGK and ES models and the HS gas as well for reference.

Table 2 shows remaining coefficients for the Shakhov, BGK, and ES models and the HS gas. There are three columns for the Shakhov model. The first column shows the values obtained by direct computations, the second those obtained by using the conversion formulas from the existing data for the BGK model, and the third those obtained by the result of the theory of symmetry relation. Fairly good agreements of the data among three columns ensure the validity of the conversion formulas, as well as the accuracy of the numerical computations in the present work.

Figure 1 shows the Knudsen-layer functions obtained from the numerical data of $\phi_{1}^{(1)}, \ldots, \phi_{8}^{(1)}, \phi_{2}^{(0)}$, and $\phi_{3}^{(0)}$. Remember that $\eta$ is the stretched coordinate normal to the boundary and that $\Omega$ 's, $\Theta$ 's, and $Y$ 's are the density, temperature, and tangential flow velocity corrections to the fluid-dynamical description inside the Knudsen layer [see (3)]. These quantities can not be recovered from the BGK model by the conversion formulas.

Finally, let us note two remarkable things. Firstly the Shakhov model does not inherit a special property of the BGK model that $c_{2}^{(0)}, \Omega_{2}^{(0)}$, and $\Theta_{2}^{(0)}$ all vanish even if $\phi_{2}^{(0)}$ does not. Secondly $c_{3}^{(0)}$ is positive irrespective of the model 
TABLE 1. Slip/jump coefficients for the Shakhov model $(\operatorname{Pr}=2 / 3)$, the BGK model, the ES model $(\operatorname{Pr}=2 / 3)$, and the HS gas. I.

\begin{tabular}{ccccccc}
\hline & $b_{1}^{(1)}$ & $b_{2}^{(1)}$ & $b_{3}^{(1)}$ & $b_{4}^{(1)}$ & $b_{5}^{(1)}$ & $b_{6}^{(1)}$ \\
\hline Shakhov & 1.01837 & 0.58736 & -1.18399 & -0.84920 & -0.66135 & 0.18785 \\
BGK [4] & 1.01619 & 0.38316 & -0.77836 & -0.76632 & -0.50002 & 0.26630 \\
ES [12] & 0.67746 & 0.38316 & -0.50025 & -0.51088 & -0.33334 & 0.17753 \\
HS [1] & 1.25395 & 0.64642 & -1.58456 & -0.90393 & -0.66012 & 0.24381 \\
\hline & $b_{7}^{(1)}$ & $b_{8}^{(1)}$ & $c_{2}^{(0)}$ & $c_{3}^{(0)}$ & $2 a_{1}^{(1)}$ & $a_{2}^{(1)}$ \\
\hline Shakhov & 0.41135 & -0.39904 & -0.29929 & 0.00966 & -0.25079 & -0.48900 \\
BGK [4] & 0.26729 & -0.26693 & 0 & 0.11168 & -0.23368 & -0.26693 \\
ES [12] & 0.20667 & -0.26693 & -0.02537 & 0.00041 & -0.15578 & -0.26693 \\
HS [1] & 0.44728 & -0.23353 & -0.49925 & 0.00874 & -0.21369 & -0.47816 \\
\hline
\end{tabular}

TABLE 2. Slip/jump coefficients for the Shakhov model $(\operatorname{Pr}=2 / 3)$, the BGK model, the ES model $(\operatorname{Pr}=2 / 3)$, and the HS gas. II.

\begin{tabular}{|c|c|c|c|c|c|c|}
\hline & \multicolumn{3}{|c|}{ Shakhov (present results) } & \multirow[b]{2}{*}{ BGK [4] } & \multirow[b]{2}{*}{ ES [12] } & \multirow[b]{2}{*}{ HS [1] } \\
\hline & direct computation & formula (10) & symmetry relation [1] & & & \\
\hline$c_{1}^{(0)}$ & 1.95408 & 1.95408 & 1.95408 & 1.30272 & 1.30160 & 2.40014 \\
\hline$c_{4}^{(0)}$ & 3.33133 & 3.33130 & 3.33130 & 1.82182 & 1.69117 & 4.61802 \\
\hline$c_{5}^{(0)}$ & 0.44046 & 0.44045 & 0.44046 & 0.44046 & 0.28954 & 0.45957 \\
\hline$c_{6}^{50)}$ & -2.14137 & -2.14140 & -2.14136 & -1.42758 & -1.35138 & -3.18002 \\
\hline
\end{tabular}

(see Table 1). The positivity leads to the theoretical prediction of the negative thermophoresis of a spherical particle which is kept at a uniform temperature (or extremely high thermal conductivity). This is the topic in the next section.

\section{APPLICATION EXAMPLE}

Consider a spherical particle of radius $L$ suspended in a slightly rarefied gas. When there is a temperature gradient in the background gas, the sphere is subjected to a force from the gas (the thermal force).

Let the origin of the space coordinates $X_{i}$ be the center of the sphere and the temperature of the gas in the absence of the sphere be $T_{0}+\left(d T / d X_{1}\right)_{\infty} X_{1}$, where $T_{0}$ is the reference temperature. When the thermal conductivity of the particle is by far larger than that of the gas, the thermal force $\left(F_{T}, 0,0\right)$ acting on the particle is given by

$$
\frac{F_{T}}{\lambda_{g} L^{2}\left(2 R T_{0}\right)^{-1 / 2}\left(d T / d X_{1}\right)_{\infty}}=24 \pi c_{3}^{(0)} \varepsilon^{2}+o\left(\varepsilon^{2}\right) .
$$

Here $\lambda_{g}$ is the thermal conductivity of the gas; see, e.g., [4, p. 252]. ${ }^{4}$ Two remarks are in order. If the thermal conductivity of the particle is comparable to that of the gas, the thermal force is larger by one order in $\varepsilon$ and is in the direction opposite to the temperature gradient. The force (11) is, however, in the same direction as the temperature gradient, because $c_{3}^{(0)}$ is positive, irrespective of the gas model (see Table 1). The former case is simply referred to as the thermophoresis, while the latter case, i.e., (11), is referred to as the negative thermophoresis. The reversal of the force direction is due to the change of the dominant physical mechanism. The reader is referred to [4] for the comprehensive explanation on this issue.

As is noted at the end of Preparation, the mean free path of different molecular models should be converted in comparing the magnitude of the thermal force $F_{T}$. In view of (11), the conversion that makes the thermal conductivity $\lambda_{g}$ (or $\gamma_{2} \varepsilon$ ) common is suitable. Adopting this conversion and neglecting the error of $o\left(\varepsilon^{2}\right)$, the magnitude of $F_{T}$ is proportional to $c_{3}^{(0)} / \gamma_{2}^{2}$, whose value is $0.00429,0.11168,0.00041$, and 0.00236 for the Shakhov, BGK, and ES models and the HS gas, respectively. Thus, the value of $F_{T}$, which involves a second order jump coefficient, depends largely on the molecular model.

\footnotetext{
${ }^{4}$ The coefficient $c_{3}^{(0)}$ is related to $a_{4}$ in [4, p. 252] as $a_{4}=-c_{1}^{(0)} b_{2}^{(1)}-b_{3}^{(1)}=(5 / 2) c_{3}^{(0)} / \operatorname{Pr}$. See (57) in [1, Sec. 7] for the last equality.
} 

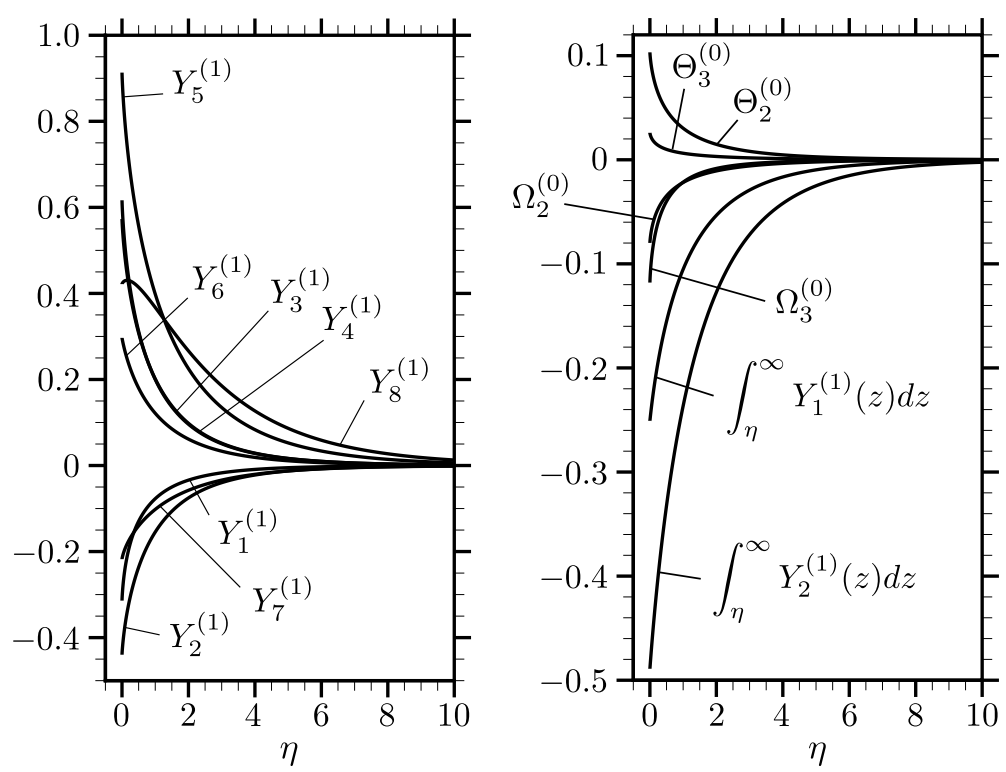

FIGURE 1. Knudsen-layer functions for the Shakhov model. Tangential velocity correction $Y_{1}^{(1)}, \ldots, Y_{8}^{(1)}$ (left) and density corrections $\Omega_{2}^{(0)}$ and $\Omega_{3}^{(0)}$, temperature corrections $\Theta_{2}^{(0)}$ and $\Theta_{3}^{(0)}$, and $\int_{\eta}^{\infty} Y_{1}^{(1)}(z) d z$ and $\int_{\eta}^{\infty} Y_{2}^{(1)}(z) d z$ (right). Note that there is a meaningful difference between the values of $Y_{3}^{(1)}$ and $Y_{4}^{(1)}$, although it is almost invisible in the figure.

\section{CONCLUSION}

In the present paper, the slip/jump coefficients and the Knudsen-layer functions for the Shakhov model occurring in the generalized slip-flow theory have been obtained up to the second order of the Knudsen number expansion. Conversion formulas from the BGK model have also been established. The thermal force in the case of negative thermophoresis has been discussed as an example together with the comparison among the different gas models.

\section{ACKNOWLEDGMENTS}

The present work is supported in part by KAKENHI from JSPS (Nos. 18K13684 and 17H03173).

\section{REFERENCES}

[1] S. Takata and M. Hattori, J. Stat. Phys. 147, 1182-1215 (2012).

[2] M. Hattori and S. Takata, Bull. Inst. Math. Acad. Sin. (N. S.) 10, 423-448 (2015).

[3] M. Hattori and S. Takata, J. Stat. Phys. 161, 1010-1036 (2015).

[4] Y. Sone, Molecular Gas Dynamics (Birkhäuser, Boston, 2007).

[5] P. L. Bhatnagar, E. P. Gross, and M. Krook, Phys. Rev. 94, 511-525 (1954).

[6] L. H. Holway, Phys. Fluids. 9, 1658-1673 (1966).

[7] E. M. Shakhov, Fluid Dyn. 3, 95-96 (1968).

[8] C. Cercignani, The Boltzmann equation and its applications, Applied Mathematical Sciences, Vol. 67 (Springer-Verlag, New York, 1988).

[9] K. Aoki, S. Takata, E. Tatsumi, and H. Yoshida, Phys. Fluids. 22, 112001-1-12 (2010).

[10] S. Takata, H. Funagane, and K. Aoki, Kinetic and Related Models 3, 353-372 (2010).

[11] H. Funagane, S. Takata, K. Aoki, and K. Kugimoto, Boll. Unione Mat. Ital. (Ser. 9) 4, 19-46 (2011).

[12] S. Takata, M. Hattori, and T. Hasebe, AIP Conf. Proc. 1786, 040004-1-8 (2016).

[13] C. E. Siewert and F. Sharipov, Phys. Fluids 14, 4123-4129 (2002).

[14] R. D. M. Garcia and C. E. Siewert, Eur. J. Mech. B/Fluids 29, 181-191 (2010).

[15] F. Sharipov, Eur. J. Mech. B/Fluids 22, 133-143 (2003).

[16] S. Takata, J. Stat. Phys. 136, 751-784 (2009).

[17] Y. Sone and Y. Onishi, J. Phys. Soc. Jpn. 44, 1981-1994 (1978). 